\title{
Toward a graph version of Rado's theorem
}

\author{
Andy Parrish \\ Department of Mathematics \\ University of California, San Diego \\ La Jolla, California, U.S.A. \\ atparrish@math.ucsd.edu
}

Submitted: Jan 24, 2013; Accepted: Feb 24, 2013; Published: Mar 1, 2013

Mathematics Subject Classifications: 05D10

\begin{abstract}
An equation is called graph-regular if it always has monochromatic solutions under edge-colorings of $K_{\mathbb{N}}$. We present two Rado-like conditions which are respectively necessary and sufficient for an equation to be graph-regular.
\end{abstract}

Keywords: coloring; graph theory; Ramsey theory; Rado's theorem

\section{Introduction}

Ramsey's theorem [3] [8] states that, given numbers $r$ and $k$, there is an $R=R(r, k)$ so that any $r$-coloring of the edges of the complete graph on $R$ vertices contains a monochromatic complete graph on $k$ vertices.

Elsewhere in Ramsey theory, the related (but seemingly-unconnected) result of Rado [2][3][7] characterizes the set of linear equations $A \mathbf{x}=\mathbf{0}$ such that, whenever $\mathbb{N}$ is finitely colored, there is a solution whose entries fall into the same color class. Such an equation is called partition-regular.

These two results were connected in a result by Deuber, Gunderson, Hindman, and Strauss [1], and followed up by Gunderson, Leader, Prömel, and Rödl [4][5]. Their results describe the equations $A \mathbf{x}=\mathbf{0}$ such that, for any $m$, a large two-colored graph must either contain a complete blue subgraph whose vertices solve the equation, or a red $K_{m}$ with no implied structure. If one can assure that there is no red $K_{m}$, then the desired structured set falls out nicely.

The first example of an equation with an unconditional monochromatic solution was given in [6]. That paper introduced the notion of a graph-regular equation. 
Definition 1. For a fixed matrix $A$ and vector $\mathbf{b}$, we say $A \mathbf{x}=\mathbf{b}$ is graph-regular if there is a function $N_{A}(r)$ so that, for all $r$, for all $N>N_{A}(r)$, every $r$-coloring of the complete graph on $[N]$ has a solution $\mathbf{x}=(x(1), \ldots, x(k))$ so that $(1)$ the edges $\{x(i), x(j)\}$ are all the same color, and (2) the values $\{x(i)\}$ are distinct.

We require a solution by distinct values due to degeneracy issues which do not appear in the case of coloring points. Further, for non-triviality, we require the equation to contain at least three variables.

As the main contribution of this paper, we give two extensions of Rado's "columns condition" to the graph setting - the weak and strong graph columns conditions. We will show that the weak version is necessary for an equation to be graph-regular, and the strong version is sufficient.

The two sets of graph columns conditions are introduced in Section 2. In Section 3, we show that the weak graph columns condition is necessary for graph-regularity, and along the way we show that $A \mathbf{x}=\mathbf{b}$ may only be graph-regular if $\mathbf{b}=\mathbf{0}$. Section 4 shows that the strong graph columns condition is sufficient for graph-regularity. Finally, in Section 5, we explore the natural extension of this problem to hypergraph-regular equations, and find that the condition is too strong - no such equation exists.

\section{The Graph Columns Condition}

\subsection{Definitions}

Rado's theorem [3][7] characterizes the partition-regular equations with use of the columns condition. Here we state a new formulation which is equivalent to the original.

Definition 2. A matrix $A$ satisfies the columns condition if there is a sequence of vectors $\mathbf{z}_{1}, \ldots, \mathbf{z}_{T}$ in the nullspace of $A$ and decreasing sequence of sets $R_{1} \supseteq \ldots \supseteq R_{T}$ so that

1. If $i \in R_{t}$, then $z_{s}(i)=0$ for all $s \leqslant t$.

2. If $i \notin R_{t}$, then there is an $s \leqslant t$ with $z_{s}(i)=1$

3. $R_{T}=\varnothing$.

Here is another perspective, closer to the usual. For a fixed $i$, the sequence of values $\left\{z_{t}(i)\right\}_{t=1}^{T}$ is restricted to being 0 until some $z_{t}(i)$ is 1 , after which it is free to take on any value. Hence the sets $R_{t}$ denote which variables are still restricted at time $t$.

The remarkable fact, proven originally in [7], and in [3], is that the columns condition determines whether an equation is regular.

Theorem 3. The equation $A \mathbf{x}=\mathbf{0}$ has a monochromatic solution under any finite coloring of $\mathbb{N}$ whenever $A$ satisfies the columns condition. If A does not satisfy the columns condition then there is some $p_{0}=p_{0}(A)$ so that, for every prime $p>p_{0}$, a monochromatic solution is avoided by the coloring $\psi_{p}$ (which will be introduced in Section 3.1). 
There are several ways to extend the columns condition to apply to edge-colorings. We state two.

Definition 4. We say a matrix $A$ with $n$ columns satisfies the weak graph columns condition (WGCC) if there is a sequence of vectors $\mathbf{z}_{0}, \ldots, \mathbf{z}_{T}$ in the nullspace of $A$, and a decreasing sequence of graphs $R_{0} \supseteq \ldots \supseteq R_{T}$ with common vertex set $[n]$ so that

1. If $\{i, j\} \in R_{t}$, then $z_{s}(i)=z_{s}(j)$ for all $s<t$.

2. If $\{i, j\} \notin R_{t}$, then there is an $s \leqslant t$ with $\left|z_{s}(j)-z_{s}(i)\right|=1$.

3. $R_{T}=\varnothing$.

4. $\mathbf{z}_{0}=1$.

Further, we say $A$ satisfies the strong graph columns condition (SGCC) if we may replace $(1)$ and $(2)$ by $\left(1^{*}\right)$ and $\left(2^{*}\right)$ :

$1^{*}$. If $\{i, j\} \in R_{t}, z_{s}(i)=z_{s}(j) \in\{0,1\}$ for all $s<t$

$2^{*}$. If $\{i, j\} \notin R_{t}$, then there is an $s \leqslant t$ with $z_{s}(i)=0$ and $z_{s}(j)=1$ (or vice versa).

In words, the $\mathbf{z}_{t}$ 's are restricted so that, for each $i, j$ pair, as $t$ increases, the values $z_{t}(i), z_{t}(j)$ are initially equal, remain equal until they differ by exactly 1 , and are unrestricted after that. An edge between $i$ and $j$ in graph $R_{t}$ means that pair remains restricted through time $t$. If $\{i, j\} \in R_{t}$, then we say the pair is restricted at time $t$, otherwise it is unrestricted.

The strong graph columns conditions requires conditions on the values $z_{t}(i)$ in addition to the differences across edges.

Example 5. Let

$$
A=\left(\begin{array}{rrrrrr}
1 & -1 & 0 & -1 & 4 & -3 \\
0 & 0 & 1 & -1 & 1 & -1
\end{array}\right)
$$

Here is one sequence of vectors showing $A$ satisfies the graph columns condition (weak and strong):

$$
\mathbf{z}_{0}=\left(\begin{array}{c}
1 \\
1 \\
1 \\
1 \\
1 \\
1
\end{array}\right) \quad \mathbf{z}_{1}=\left(\begin{array}{c}
1 \\
1 \\
0 \\
0 \\
0 \\
0
\end{array}\right) \quad \mathbf{z}_{2}=\left(\begin{array}{c}
1 \\
0 \\
1 \\
1 \\
0 \\
0
\end{array}\right) \quad \mathbf{z}_{3}=\left(\begin{array}{c}
3 \\
0 \\
1 \\
0 \\
0 \\
1
\end{array}\right)
$$

The corresponding restriction graphs may be described simply:

- $R_{0}$ : All edges are restricted $-R_{0}=K_{6}$.

- $R_{1}$ : All edges among $\{1,2\}$ and $\{3,4,5,6\}$ remain restricted. 
- $R_{2}$ : Edges $\{3,4\}$ and $\{5,6\}$ remain restricted.

- $R_{3}$ is empty - no edges are restricted.

Note that, at each step, $R_{t}$ is a union of disjoint cliques. This always happens.

Example 6. Fix any nonzero $r \in Q$. Let

$$
A=\left(\begin{array}{rrrrrrcc}
1 & -1 & 0 & 0 & 0 & 0 & -1 & 1 \\
0 & 0 & 1 & -1 & 0 & 0 & -1 & 1 \\
0 & 0 & 0 & 0 & 1 & -1 & -1 & 1 \\
0 & -1 & 0 & -1 & 0 & -1 & -r & r+1
\end{array}\right) .
$$

Here is one sequence of vectors showing $A$ satisfies the weak graph columns condition:

$$
\mathbf{z}_{0}=\left(\begin{array}{c}
1 \\
1 \\
1 \\
1 \\
1 \\
1 \\
1 \\
1
\end{array}\right) \quad \mathbf{z}_{1}=\left(\begin{array}{c}
1 \\
1 \\
1 \\
1 \\
0 \\
0 \\
0 \\
0
\end{array}\right) \quad \mathbf{z}_{2}=\left(\begin{array}{c}
1 \\
1 \\
0 \\
0 \\
1 \\
1 \\
0 \\
0
\end{array}\right) \quad \mathbf{z}_{3}=\left(\begin{array}{c}
1 \\
0 \\
1 \\
0 \\
1 \\
0 \\
r+1 \\
r
\end{array}\right)
$$

Notice that $\mathbf{z}_{3}$ relaxes the restriction on the 7th and 8th columns by using values $r$ and $r+1$, rather than 0 and 1 as required by the strong graph columns condition. Indeed, $A$ satisfies WGCC, but fails to satisfy SGCC. We believe 8 variables is minimal to separate these conditions.

We now state our main result:

Theorem 7. Fix a matrix A. If Ax $=\mathbf{0}$ is graph-regular, then $A$ satisfies the weak graph columns condition. If $A$ satisfies the strong graph columns condition, then $A \mathbf{x}=\mathbf{0}$ is graph-regular.

We will prove WGCC is necessary in Section 3. The proof is based on both the techniques and result of Rado, and is not particularly deep. In Section 4, we will show SGCC is sufficient. The proof uses the machinery from [6] to expand the family of known graph-regular equations which were missed on the first pass.

Of course the most interesting question here is to close the gap between the strong and weak conditions - to classify the remaining equations. Is the matrix from Example 6 graph-regular?

\section{$3 \quad$ Necessary conditions for graph-regularity}

In this section, we define the appropriate colorings which allow us to essentially repeat the proof of Rado's theorem for our setting. After these colorings show some initial structure of graph-regular equations, we will apply Rado's theorem to show that WGCC is necessary. 


\subsection{Coefficients must add to 0}

We define a family of colorings, $f_{n}$, of $\left(\begin{array}{c}\mathbb{N} \\ 2\end{array}\right)$ by

$$
f_{n}(a n+x, b n+y)= \begin{cases}\text { blue } & \text { if } x=y \\ \min \{x, y\} & \text { if } x \neq y\end{cases}
$$

where $x, y \in\{0,1, \ldots, n-1\}$, and where we storm past the usual boundary between actual colors and mathematician's colors. We claim that all monochromatic triangles under $f_{n}$ are blue.

Consider a triangle $\{x, y, z\}$ with no blue edge, where $x=a n+i, y=b n+j, z=c n+k$, and $i, j, k \in\{0,1, \ldots, n-1\}$. Then the numbers $i, j, k$ must be distinct. Reordering so that $i<j<k$, we see that $f_{n}(x, y)=f_{n}(x, z)=i$, while $f_{n}(y, z)=j \neq i$. Thus a triangle without a blue edge cannot be monochromatic. Turning this around, any monochromatic triangle must be blue.

Going back to the definition of $f_{n}$, this means that any monochromatic triangle and hence any monochromatic clique - must represent only one congruence class mod $n$.

Lemma 8. If $\sum a_{i} x_{i}=b$ is graph-regular with $b, a_{i} \in \mathbb{Z}$, then $b$ is a multiple of $\sum a_{i}$.

Proof. Write $\sum a_{i}=M$. If $M \neq 0$, then consider the coloring $f_{M}$. Let $\left\{x_{i}\right\}$ be monochromatic under $f_{M}$, so that $x_{i}=b_{i} M+c$.

Then we have

$$
\begin{aligned}
\sum a_{i} x_{i} & =\sum a_{i}\left(b_{i} M+c\right) \\
& =\left(\sum a_{i} b_{i} M\right)+\sum a_{i} c \\
& =\left(\sum a_{i} b_{i}\right) M+c M .
\end{aligned}
$$

We see that $\sum a_{i} x_{i}$ is a multiple of $M$. If $\sum a_{i} x_{i}=b$, then we see that $b$ is a multiple of $M$ as well.

On the other hand, if $M=0$, then we may repeat the above argument using any $f_{n}$. Since the $c M$ term goes away, we learn that $b$ is a multiple of $n$ for every $n$ we choose, forcing $b=0$ as well.

Fix $n$, and define the coloring $g_{n}$ of $\left(\begin{array}{c}\mathbb{N} \\ 2\end{array}\right)$ by

$$
g_{n}\left(n^{j} a, n^{k} b\right)= \begin{cases}\text { red } & \text { if } j \neq k \\ f_{n}(a, b) & \text { if } j=k,\end{cases}
$$

where $a$ and $b$ are not divisible by $n$.

Note 9 . Using the same argument as for $f_{n}$, we see that any triangle which is monochromatic under $g_{p}$ must be red or blue for $p$ prime. Writing $x_{i}=b_{i} n^{r_{i}}$, this means either all $r_{i}$ values are distinct (yielding a red clique), or all $r_{i}$ values are equal and all $b_{i}$ values are congruent modulo $n$ (yielding a blue clique).

Lemma 10. If $\sum a_{i} x_{i}=b$ is graph-regular with $b, a_{i} \in \mathbb{Z}$, then $\sum a_{i}=b=0$. 
Proof. Suppose $\sum a_{i} x_{i}=b$ is graph-regular, with $M=\sum a_{i} \neq 0$. By Lemma 8, we may write $b=k M$. We assume each $a_{i}$ is non-zero, as removing superfluous variables will preserve graph-regularity.

We apply a new coloring, which should be thought of as a hybrid between the colorings $f_{n}$ and $g_{n}$. There is a prime $p$ which does not divide $M$ nor any of the $a_{i}$ values, since none of these values is 0 . For any $x$, we may uniquely write $x=c p+d+k$, where $d \in\{0,1, \ldots, p-1\}$ and $k=\frac{b}{M}$ was defined above.

Using this form, we define

$$
\chi\left(c p+d+k, c^{\prime} p+d^{\prime}+k\right)= \begin{cases}\min \left\{d, d^{\prime}\right\} & \text { if } d \neq d^{\prime} \\ g_{p}\left(c, c^{\prime}\right) & \text { if } d=d^{\prime}\end{cases}
$$

Note that we treat the colors from the two pieces of this function as distinct - all pairs in a monochromatic clique must have either all used the first piece, or all used the second. In fact, we have already seen from our analysis of $f_{n}$ that no monochromatic clique can arise from the first piece of the definition of $\chi$, so any solution must come from $g_{p}$.

Let $\left\{x_{i}\right\}$ be a monochromatic solution. Since all edges must have been colored by the second piece of $\chi$, we can write $x_{i}=\beta_{i} p+d+k$, where $d \in\{0,1, \ldots, p-1\}$ is common for each $x_{i}{ }^{1}$ This gives us

$$
\begin{aligned}
\sum a_{i} x_{i} & =b \\
\sum a_{i}\left(\beta_{i} p+d+k\right) & =k M \\
\sum a_{i} \beta_{i} p+d M+k M & =k M \\
\left(\sum a_{i} \beta_{i}\right) p+d M & =0 .
\end{aligned}
$$

Since $p$ does not divide $M$, and $d$ is less than $p$, we must have $d=0$. Dividing by $p$, we are left with

$$
\sum a_{i} \beta_{i}=0
$$

Write $\beta_{i}=\left(b_{i} p+c_{i}\right) p^{r_{i}}$, with $c_{i} \in\{1,2, \ldots, p-1\}$. From Note 9 , we have either a red clique with each $r_{i}$ distinct, or we have a blue clique with $r_{i}=r$ and $c_{i}=c$ common across all $i$.

Case 1. The clique is blue, so $\beta_{i}=\left(b_{i} p+c\right) p^{r}$.

We see that

$$
\begin{aligned}
\sum a_{i}\left(b_{i} p+c\right) p^{r} & =0 \\
\sum a_{i}\left(b_{i} p+c\right) & =0 \\
\sum a_{i} c & \equiv 0 \quad(\bmod p) \\
c M & \equiv 0 \quad(\bmod p) .
\end{aligned}
$$

Since $p$ divides neither $c$ nor $M$, this is impossible.

Case 2. The clique is red, so $\beta_{i}=\left(b_{i} p+c_{i}\right) p^{r_{i}}$, with each $r_{i}$ distinct.

\footnotetext{
${ }^{1}$ For small values of $x_{i}$, the resulting $\beta_{i}$ may be zero or negative. A little care is required to handle $\beta_{i}=0$, but we will ignore it here.
} 
Let $r_{j}$ be the unique smallest exponent. We find

$$
\begin{aligned}
\sum a_{i}\left(b_{i} p+c_{i}\right) p^{r_{i}} & =0 \\
\sum a_{i}\left(b_{i} p+c_{i}\right) p^{r_{i}-r_{j}} & =0 \\
a_{j}\left(b_{j} p+c_{j}\right)+\sum_{i \neq j} a_{i}\left(b_{i} p+c_{i}\right) p^{r_{i}-r_{j}} & =0 \\
a_{j} c_{j} & \equiv 0 \quad(\bmod p)
\end{aligned}
$$

Again, since $p$ divides neither $c$ nor $a_{j}$, this is impossible.

Together, we have seen that $M \neq 0$ is impossible, so $\sum a_{i}=M=0$. Since $b=k M$, we also get $b=0$ for free.

Note that Lemma 10 extends to systems of linear equations - if $A \mathbf{x}=\mathbf{b}$ is graphregular, then $\mathbf{b}=\mathbf{0}$ and the columns of $A$ sum to $\mathbf{0}$. This is easily seen since each equation from the system $A \mathbf{x}=\mathbf{b}$ must also be graph-regular.

Consider such an equation, $\mathbf{a}_{1} x_{1}+\ldots+\mathbf{a}_{k} x_{k}=0$, where the coefficients sum to 0 . We may rewrite this as, for instance,

$$
\mathbf{a}_{1}\left(x_{1}-x_{k}\right)+\ldots+\mathbf{a}_{k-1}\left(x_{k-1}-x_{k}\right)=0
$$

now an equation relating differences. This suggests that we should consider colorings based on these differences - colorings of the form $\chi(x<y)=f(y-x)$. We may now take guidance from Rado's theorem to get a better handle on things.

For a prime $p$, and $x=p^{r}(b p+s)$, let $\psi_{p}(x)=s \in[p-1]$ be the "super $\bmod p$ " coloring, from Rado's theorem. Rado's theorem suggests to us that, when looking at colorings based only on differences between endpoints, we need only consider the colorings $\psi_{p}$. We will show that Rado's theorem does apply here, but we begin with a simple consequence to give a feel for how it works.

Theorem 11. Let $\sum_{i=1}^{k} a_{i} x_{i}=0$ be graph-regular with $a_{i} \in \mathbb{Z}$. Then there is a nonempty set $I \subsetneq[k]$ so that

$$
\sum_{i \in I} a_{i}=\sum_{j \notin I} a_{j}=0 .
$$

To prove this, we introduce the graph version of $\psi_{p}$.

Define $\varphi_{p}:\left(\begin{array}{c}\mathbb{N} \\ 2\end{array}\right) \rightarrow[p-1]$ by

$$
\varphi_{p}(x<y)=\psi_{p}(y-x) .
$$

Proof. Fix a prime $p$ and color $\left(\begin{array}{c}\mathbb{N} \\ 2\end{array}\right)$ by $\varphi_{p}$. Suppose $x_{1}, \ldots, x_{k}$ are distinct values satisfying $a_{1} x_{1}+\ldots+a_{k} x_{k}=0$, with the edges among them all color $c$. Let $x_{j}$ be the smallest of these values. As noted earlier, we see that

$$
\sum_{i \neq j} a_{i}\left(x_{i}-x_{j}\right)=0 .
$$


By choice of $x_{j}$, each of the terms $x_{i}-x_{j}$ is positive. Thus we may write $x_{i}-x_{j}=$ $p^{r_{i}}\left(b_{i} p+c\right)$, since $\varphi_{p}\left(x_{j}<x_{i}\right)=\psi_{p}\left(x_{i}-x_{j}\right)=c$. Let $r$ be the smallest exponent among these $k-1$ terms, and let $I=\left\{i \in[k] \backslash\{j\} \mid r_{i}=r\right\}$. Note that $\varnothing \subsetneq I \subsetneq[k]$. We see that

$$
\begin{aligned}
0 & =\sum_{i \neq j} a_{i} p^{r_{i}}\left(b_{i} p+c\right) \\
& =\sum_{i \neq j} a_{i} p^{r_{i}-r}\left(b_{i} p+c\right) \\
& =\sum_{i \in I} a_{i}\left(b_{i} p+c\right)+p\left(\sum_{i \notin I \cup\{j\}} a_{i} p^{r_{i}-r-1}\left(b_{i} p+c\right)\right) \\
& \equiv c\left(\sum_{i \in I} a_{i}\right) \quad(\bmod p)
\end{aligned}
$$

Since $c$ is in $[p-1]$, we see that $p$ divides $\sum_{i \in I} a_{i}$. If we take $p>\sum_{i=1}^{k}\left|a_{i}\right|$, then the only way this can happen is if $\sum_{i \in I} a_{i}=0$. Since we already know that $\sum_{i=1}^{k} a_{i}=0$, we learn that $\sum_{j \notin I} a_{j}=0$ as well.

Corollary 12. No nondegenerate homogeneous linear equation of three variables is graphregular.

This was already proven in [6] using Brooks' theorem, but we give a simpler proof.

Proof. Let $k=3$, and let $I \subsetneq\{1,2,3\}$ be nonempty. Then either $I$ or its complement has a single element. The corresponding coefficient must be 0 , meaning the equation depends on at most two variables and is trivial.

Corollary 13. Up to rescaling, the only graph-regular homogeneous linear equation of four variables is $w-x+y-z=0$.

That this equation is regular was shown in [6]. We show that it stands alone.

Proof. Let $a w+b x+c y+d z=0$ be graph-regular, with $a, b, c, d \in \mathbb{Z}_{\neq 0}$. By Theorem 11 , we know that two complementary subsets of the coefficients must add to zero. Up to permutation, this leaves us with $a w-a x+c y-c z=0$, or rather $a(w-x)=c(z-y)$. We may assume both $a$ and $c$ are positive by switching $w$ and $x$, or $y$ and $z$. We claim $a=c$.

Suppose not. After canceling common factors, we may assume we may assume $c$ is divisible by some prime $p$ which does not divide $a$. Pick $r$ so that $p^{r}$ divides $c$, but $p^{r+1}$ does not. Consider the 2-coloring given by

$$
\chi(x, y) \equiv\left\lfloor\frac{f(x, y)}{r}\right\rfloor \quad(\bmod 2)
$$

where $f(x, y)$ gives the highest exponent of $p$ which divides $x-y$.

Now suppose $a(w-x)=c(z-y)$, with $w, x, y, z$ distinct. Let $f(w, x)=k$. This means that $a(w-x)$ represents a power of $p^{k}$ on the left hand side. Dividing by $c$, we learn that 
$(z-y)$ represents a power of $p^{k-r}$, so $f(y, z)=k-r$. Looking at the corresponding $\chi$ values of $\{w, x\}$ and $\{y, z\}$, we see that they are different, so the edges among $\{w, x, y, z\}$ are not monochromatic.

\subsection{Considering $\varphi_{p}$}

Lemma 14. Let $A$ be a matrix whose columns sum to $\mathbf{0}$. If the equation $A \mathbf{x}=\mathbf{0}$ has a monochromatic solution under the edge-coloring $\varphi_{p}$ for every prime $p$, then $A$ satisfies the weak graph columns condition.

Proof. Let $A \mathbf{x}=\mathbf{0}$ have a monochromatic solution under $\varphi_{p}$ for every prime $p$. Denote the columns of $A$ by $\left\{\mathbf{a}_{i}\right\}_{i=1}^{n}$.

From $A$, we will make a larger matrix $C$ with columns indexed by $\left(\begin{array}{c}{[n]} \\ 2\end{array}\right)=\{(i, j) \mid 1 \leqslant$ $i<j \leqslant n\}$. The columns of $C$ come from gluing the columns of $A$ (or the zero vector) to new vectors which bind relationships between the columns of $A$.

$$
\mathbf{c}_{1 j}=\left(\begin{array}{c}
\mathbf{a}_{j} \\
- \\
\mathbf{b}_{1 j}
\end{array}\right), \text { and } \mathbf{c}_{i j}=\left(\begin{array}{c}
\mathbf{0} \\
- \\
\mathbf{b}_{i j}
\end{array}\right) \text { if } i>1,
$$

where

$$
b_{i j}(k, \ell)= \begin{cases}1 & \text { if }(k, \ell)=(1, j) \\ -1 & \text { if }(k, \ell)=(1, i) \text { or }(i, j) \\ 0 & \text { otherwise }\end{cases}
$$

Note that the matrix $C$ does not explicitly contain the column $\mathbf{a}_{1}$. However, since $\sum \mathbf{a}_{i}=\mathbf{0}$, that information is not lost.

Suppose $C \mathbf{y}=\mathbf{0}$, with $y(1, j)=x(j)-x(1)$. The vectors $\left\{b_{i j}\right\}$ are designed so that $y(i, j)=x(j)-x(i)$.

Turned around, when $A \mathbf{x}=\mathbf{0}$, and $\mathbf{y}$ is defined above, we get $C \mathbf{y}=\mathbf{0}$. Likewise, if $C \mathbf{y}=\mathbf{0}$ then, for any value $x(1)$, the values $x(i)$ are uniquely defined from $\mathbf{y}$, and they satisfy $A \mathbf{x}=\mathbf{0}$.

We would like to say that, when $A \mathbf{x}=\mathbf{0}$ is a monochromatic solution under the edgecoloring $\varphi_{p}$, the corresponding solution to $C \mathbf{y}=\mathbf{0}$ is monochromatic under the vertexcoloring $\psi_{p}$. However, this is not quite true. The definition says $\varphi_{p}(x, y)=\psi_{p}(y-x)$ only when $x<y$. For a monochromatic solution under $\psi_{p}$, we would need $x(1)<x(2)<$ $\ldots<x(n)$. Instead, for each permutation $\sigma \in S_{n}$, we must define the matrix $C(\sigma)$ which will "work" when $x\left(\sigma_{1}\right)<x\left(\sigma_{2}\right)<\ldots<x\left(\sigma_{n}\right)$. We omit the definition of $C(\sigma)$, but it is essentially the same as $C$, defined in such a way that $y(i, j)$ is always a positive number when $\mathbf{x}$ is ordered by $\sigma$.

If $\mathbf{x}$ is a solution to $A \mathbf{x}=\mathbf{0}$, with $x\left(\sigma_{1}\right)<x\left(\sigma_{2}\right)<\ldots x\left(\sigma_{n}\right)$, then there is a corresponding solution to $C(\sigma) \mathbf{y}=\mathbf{0}$ by positive numbers, where $y(i, j)=x(\sigma(j))-x(\sigma(i))$. When $\mathbf{x}$ is monochromatic under $\varphi_{p}, \mathbf{y}$ is monochromatic under $\psi_{p}$.

We claim that some $C(\sigma)$ satisfies the columns condition. Indeed, by Rado's theorem, any $C(\sigma)$ failing to satisfy the columns condition has a monochromatic solution to 
$C(\sigma) \mathbf{y}=\mathbf{0}$ under $\psi_{p}$ for only finitely many primes $p$. Since each $p$ yields some $\sigma$ for which there is a solution, we learn that some $C(\sigma)$ gives a solution for infinitely many values of $p$. That $C(\sigma)$ must satisfy the columns condition.

Fix such a $\sigma$. For simplicity, we reorder the columns of $A$ so that $\sigma$ is the identity, and $C(\sigma)$ is the matrix $C$ described originally.

The columns condition gives us vectors $\mathbf{w}_{1}, \ldots, \mathbf{w}_{T}$ indexed by $\left(\begin{array}{c}{[n]} \\ 2\end{array}\right)$, and sets $R_{1} \supseteq$ $\ldots \supseteq R_{T}=\varnothing$ with vertex set $\left(\begin{array}{c}{[n]} \\ 2\end{array}\right)$ satisfying conditions (1)-(3) of Definition 2.

Define a sequence of vectors $\mathbf{z}_{1}, \ldots, \mathbf{z}_{T}$ on $[n]$ by $z_{t}(1)=0$, and $z_{t}(i)=w_{t}(1, i)$ for $i>1$. Additionally define $\mathbf{z}_{0}=\mathbf{1}$ and $R_{0}=\left(\begin{array}{c}{[n]} \\ 2\end{array}\right)$. We just need $\left\{\mathbf{z}_{t}\right\},\left\{R_{t}\right\}$ to satisfy requirements (1)-(4) of the graph columns condition.

It will be helpful to know that, for $k<\ell$,

$$
z_{t}(\ell)-z_{t}(k)=w_{t}(k, \ell)
$$

To see this, consider the $\{k, \ell\}$ row of the vectors $\mathbf{b}_{i j}$ within $C$. Since $C \mathbf{w}=\mathbf{0}$, inspecting this row tells us that

$$
w_{t}(1, \ell)-w_{t}(1, k)=w_{t}(k, \ell) .
$$

By definition of $\mathbf{z}_{t}$, we see that

$$
z_{t}(\ell)-z_{t}(k)=w_{t}(k, \ell)
$$

as desired.

Using this equation, properties (1)-(3) are immediate. Property (4) comes from the assumption that the columns of $A$ sum to $\mathbf{0 .}$

Corollary 15. If a matrix $A$ is graph-regular, then it satisfies the weak graph columns condition.

Proof. From Lemma 10, we know that the columns of $A$ sum to $\mathbf{0}$. Since $A$ is graphregular, it must have a monochromatic solution under $\varphi_{p}$ for every prime $p$. By Lemma 14, $A$ satisfies the weak graph columns condition.

We end this section by considering the sufficiency of the WGCC.

Corollary 16. If a matrix A satisfies the weak graph columns condition but is not graphregular, then the offending coloring is not of the form $\chi(x<y)=f(y-x)$.

To see this in action, consider the coloring $\varphi_{p}$ and the matrix $A$ from Example 6 . Suppose that $1<r<p^{k}-1$. Consider the vector $\mathbf{x}$ in the nullspace of $A$ given by

$$
\mathbf{x}=\left(p^{k+2}+1\right) \mathbf{z}_{1}+\left(p^{k+1}+p\right) \mathbf{z}_{2}+p^{2} \mathbf{z}_{3}
$$

where $\left\{\mathbf{z}_{i}\right\}$ come from the analysis of this example earlier. It is easy to check that $x(1)>x(2)>\ldots>x(8)$, and that $\varphi_{p}$ colors all edges by "1". Indeed, Lemma 14 actually shows that all colorings based on the difference of the endpoints will yield a monochromatic solution. Therefore, if the equation $A \mathbf{x}=\mathbf{0}$ is not graph-regular, it must be from some other type of coloring. 


\section{Sufficient conditions for graph-regularity}

We now prove the strong graph columns condition. We only have one tool at our disposal to actually find a monochromatic solution to a general edge coloring - the machinery from [6]. In order to get more mileage out of it, we define a large, hierarchical parameterized grid, which we call a rrid $_{n}$. The details of the definition are chosen to capture all of the monochromatic structure guaranteed in that paper.

Definition 17. Fix $\mathbf{x}, \mathbf{y}, \mathbf{b}, \mathbf{d} \in \mathbb{N}^{n}$. We say the grid of depth $n$ with parameters $\mathbf{x}, \mathbf{y}, \mathbf{b}, \mathbf{d}$ - abbreviated $\operatorname{Grid}_{n}(\mathbf{x}, \mathbf{y}, \mathbf{b}, \mathbf{d})$ - is the collection of points $A=\bigcup_{k=1}^{n} A_{k}$ where $A_{k}$ is the set of points of the form

$$
\left(\sum_{\ell=1}^{k-1} h(\ell) d(\ell)+x(k) d(k)+i d(k+1), \sum_{\ell=1}^{k-1} h(\ell) d(\ell)+y(k) d(k)+j d(k+1)\right)
$$

such that $h(\ell) \in\{x(\ell), y(\ell)\}$ and $i, j \in[-c(k), c(k)]$ for some $c(k) \geqslant b(k)$. As it is not earlier defined, we use $d(n+1)=0$.

For such a grid to behave nicely, we always require the parameters to satisfy:

1. $d(k)$ divides $d(k+1)$,

2. $x(k) b(k), y(k) b(k) \leqslant c(k-1)$.

As a technical note, the use of $c(k)>b(k)$ comes from requirement (2).

We say that a $\operatorname{Grid}_{n}(\mathbf{x}, \mathbf{y}, \mathbf{b}, \mathbf{d})$ is "proper" if (a) all $x$-coordinates of each of its points is less than all $y$-coordinates, and (b) each coordinate has a unique representation of the form

$$
\sum_{\ell=1}^{k} h(\ell) d(\ell)+i d(k+1)
$$

over all values of $k \in[n], h(\ell) \in\{x(\ell), y(\ell)\}$, and $i, j \in[-c(k), c(k)]$. We may thus unambiguously say that a point "resides at the $k^{t h}$ level" of the grid if its coordinates agree on $h(\ell)$ for all $\ell<k$, but disagree on $h(k)$.

For convenience of notation, we will treat a proper Grid $_{n}$ as a graph, since it uniquely stores pairs $\{x, y\}$.

The definition of a rrid $_{n}$ is admittedly overwhelming; It is best to think hierarchically. A Frid $_{1}$ (or the $A_{1}$ portion of a $\left.G r i d_{n}\right)$ is simply a square grid of lattice points $(x+i d, y+$ $j d)$ for $i, j \in[-c, c]$. The value $c$ is chosen to be at least the prescribed minimum bound b. A Grid $_{1}$ is exactly what is guaranteed monochromatic by the Gallai-Witt theorem [2][3]. The definition here appears slightly more general, but nothing is lost by assuming $d(1)=1$. When we view points as the edges of a graph, this grid will correspond to a complete bipartite graph between two sets of arithmetic progressions - the vertices $\{x+i d\}$ on one side, and $\{y+j d\}$ on the other.

A Grid $_{2}$ extends a Grid $_{1}$, and is given by $A_{1} \cup A_{2}$. Beyond the Grid $_{1}$, it includes two additional grids of the same type: one with points of the form $\left(x+x^{\prime} d+i d^{\prime}, x+y^{\prime} d+j d^{\prime}\right)$, 
and the other with points $\left(y+x^{\prime} d+i d^{\prime}, y+y^{\prime} d+j d^{\prime}\right)$ where $d^{\prime}$ is a multiple of the original $d$. As before, each of these grids correspond to complete bipartite graphs between two arithmetic progressions. Better, because of points (1) and (2) above, in each case the two

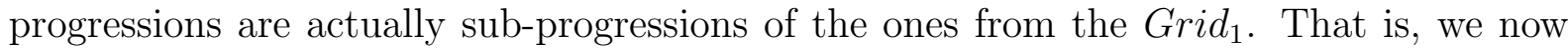
have four progressions - anchored at $x+x^{\prime} d, x+y^{\prime} d, y+x^{\prime} d$, and $y+y^{\prime} d$ respectively - so that the complete 4-partite graph among them is contained in the $\mathrm{Grid}_{2}$.

Continuing this logic, a rrid $_{n}$ includes a complete $2^{n}$-partite graph on arithmeticallyrelated points, as well as some other edges.

The proof of Theorem 3.1 in [6] may be easily modified to give the following lemma.

Lemma 18. Fix $\mathbf{b} \in \mathbb{N}^{n}$. There is a number $Q=Q(r, \mathbf{b})$ so that every $r$-coloring of $[Q]^{2}$ admits vectors $\mathbf{x}, \mathbf{y}, \mathbf{d} \in \mathbb{N}^{n}$ such that $\operatorname{Grid}_{n}(\mathbf{x}, \mathbf{y}, \mathbf{b}, \mathbf{d})$ is proper and monochromatic.

Moreover, the dependencies are such that the value of $b(k)$ may be a function of upper bounds for $x(k+1), y(k+1), c(k+1)$, and $\frac{d(k+1)}{d(k)}$.

This lemma tells that we can always find a "large" monochromatic Grid ${ }_{n}$.

\subsection{A Grid $_{n}$ is enough}

Since we know every finite-coloring of $[Q] \times[Q]$ contains a large monochromatic Grid $_{n}$ (for $Q$ sufficiently large), we only need to show the following.

Lemma 19. Let A satisfy the strong graph columns condition. Then there is some $n$, b so that the following holds. For every every proper $G=G_{r i d}(\mathbf{x}, \mathbf{y}, \mathbf{b}, \mathbf{d})$, there is a solution to $A \mathbf{w}=\mathbf{0}$ so that, for all $i, j$, the edge $\{w(i), w(j)\}$ is in $G$.

In particular, if $A$ satisfies the strong graph columns condition in $T$ steps, then we may take $n=T$.

Proof. Let $A$ satisfy the columns condition, by vectors $\mathbf{z}_{0}=\mathbf{1}, \mathbf{z}_{1}, \ldots, \mathbf{z}_{T}$ and graphs $R_{0} \supseteq \ldots \supseteq R_{T}=\varnothing$.

Fix $\mathbf{x}, \mathbf{y}, \mathbf{d} \in \mathbb{N}^{T}$. Define a sequence of vectors by

$$
\mathbf{v}_{t}=x(t) d(t) \mathbf{z}_{0}+(y(t)-x(t)) d(t) \mathbf{z}_{t}=x(t) d(t) \mathbf{1}+(y(t)-x(t)) d(t) \mathbf{z}_{t},
$$

each in the nullspace of $A$.

Define $\mathbf{w}=\sum_{t=1}^{T} \mathbf{v}_{t}$. As a sum of vectors in the nullspace of $A$, we have $A \mathbf{w}=\mathbf{0}$. We claim that this is the desired solution. It remains to show show that (1) every edge $\{w(i), w(j)\}$ is in $G$, and (2) the values $w(i)$ are distinct.

Fix two indices $i$ and $j$. By the strong columns condition, we know that the values $z_{t}(i)$ and $z_{t}(j)$ are initially equal — with common value 0 or 1 - when $\{i, j\} \in R_{t}$. There is a first time $t^{*}$ such that (without loss of generality) $z_{t^{*}}(i)=0$ and $z_{t^{*}}(j)=1$.

Moving to $\mathbf{w}$, we see that $z_{t}(i)=0$ contributes $x(t)$ to $w(i)$, and $z_{t}(i)=1$ contributes $y(t)$ to $w(i)$. Since $v_{t}(i)$ and $v_{t}(j)$ agree for $t<t^{*}$, we may call the common contribution 
at the $t$ step $h(t)=x(t)$ or $y(t)$. At time $t^{*}, v(i)=x\left(t^{*}\right)$ while $v(j)=y\left(t^{*}\right)$. This suggests the edge $\{w(i), w(j)\}$ should reside at the $\left(t^{*}\right)^{t h}$ level of $G$. For $t>t^{*}$, we have

$$
\begin{aligned}
\left|v_{t}(i)\right| & =\left|x(t) d(t)+(y(t)-x(t)) d(t) z_{t}(i)\right| \\
& =\left|x(t)+(y(t)-x(t)) z_{t}(i)\right| \times d(t) \\
& \leqslant\left|y(t)+y(t) z_{t}(i)\right| \times d(t) \quad \text { since } 0 \leqslant x(t) \leqslant y(t) \\
& \leqslant\left(\left\|z_{t}\right\|_{\infty}+1\right) y(t) d(t)
\end{aligned}
$$

Thus we see the total contribution to $w(i)$ from the tail of the sum comes to

$$
\begin{aligned}
\left|\sum_{s>t^{*}} v_{t}(i)\right| \leqslant & \sum_{s>t^{*}}\left(\left\|z_{s}\right\|_{\infty}+1\right) y(s) d(s) \\
\leqslant & \left(\left\|z_{t^{*}+1}\right\|_{\infty}+1\right) y\left(t^{*}+1\right) d\left(t^{*}+1\right)+\ldots+ \\
& +\left(\left\|z_{T-1}\right\|_{\infty}+1\right) y(T-1) d(T-1)+\left(\left\|z_{T}\right\|_{\infty}+1\right) y(T) d(T)
\end{aligned}
$$

We now begin to see an appropriate choice of $\mathbf{b}$. Set

$$
b(T) \geqslant\left\|z_{T}\right\|_{\infty}+1
$$

The last term of our bound above becomes $b(T) y(T) d(T)$, which by assumption is less than $c(T-1) d(T)$.

Next take

$$
b(T-1) \geqslant\left(\left\|z_{T-1}\right\|_{\infty}+1\right) y(T-1)+c(T-1)\left(\frac{d(T)}{d(T-1)}\right) .
$$

Now the last two terms of the sum are bounded by $c(T-2) d(T-1)$.

We may continue this process so that, for $t>t^{*}$, we have

$$
b(t) \geqslant\left(\left\|z_{t}\right\|_{\infty}+1\right) y(t)+c(t)\left(\frac{d(t+1)}{d(t)}\right) .
$$

Working backwards to step $t^{*}+1$, we get

$$
\left|\sum_{t>t^{*}} v_{t}(i)\right| \leqslant c\left(t^{*}\right) d\left(t^{*}+1\right) .
$$

We have written

$$
\begin{aligned}
w(i) & =h(1) d(1)+\ldots+h\left(t^{*}-1\right) d\left(t^{*}-1\right)+x\left(t^{*}\right) d\left(t^{*}\right)+p d\left(t^{*}+1\right) \\
w(j) & =h(1) d(1)+\ldots+h\left(t^{*}-1\right) d\left(t^{*}-1\right)+y\left(t^{*}\right) d\left(t^{*}\right)+q d\left(t^{*}+1\right)
\end{aligned}
$$


with $p, q \in\left[-c\left(t^{*}\right), c\left(t^{*}\right)\right]$. Thus we see $\{w(i), w(j)\}$ is indeed a point in $G$, in the $\left(t^{*}\right)^{t h}$ level.

To wrap up this argument, we need only take $b(t)$ to be the maximum of the lower bounds seen, over all choices of $i$ and $j$.

Finally, showing the points are distinct is simple. Since $z_{t^{*}}(i)=0$ and $z_{t^{*}}(j)=1$, we see that $w(i)$ involves $x\left(t^{*}\right)$, while $w(j)$ involves $y\left(t^{*}\right)$. Since the grid is proper, the values $w(i)$ and $w(j)$ must be distinct.

Corollary 20. Let A satisfy the strong graph columns condition. Then $A \mathbf{x}=\mathbf{0}$ is graphregular.

Proof. Let $\mathbf{c}$ be the vector given in Lemma 19, and let $r \in \mathbb{N}$ be a number of colors. We claim that, if $Q \geqslant Q(r, \mathbf{c})$ from Lemma 18, then any $r$-coloring of $\left(\begin{array}{c}{[Q]} \\ 2\end{array}\right)$ will contain a solution to $A \mathbf{x}=\mathbf{0}$ so that the values $\{x(i)\}$ are distinct, and the edges $\{x(i), x(j)\}$ are monochromatic.

Indeed, by Lemma 18, viewing $\chi$ as an $r$-coloring of $[Q] \times[Q]$ (minus the diagonal),

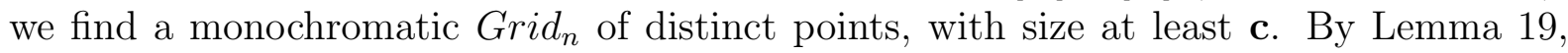
this Grid $_{n}$ contains a solution to $A \mathbf{x}=\mathbf{0}$ as desired.

\section{$5 \quad$ Hypergraph-regular equations}

There is a natural extension of graph-regularity to the hypergraph Ramsey theorem.

Unfortunately, this extension is not fruitful. Say a homogeneous linear equation is " $r$ graph-regular" if, for every coloring of the $r$-sets of $\mathbb{N}$, it has a monochromatic solution by distinct numbers. As with graphs, when considering an $r$-uniform hypergraph, we require the equations to have at least $r+1$ variables, or else every solution will be trivially monochromatic.

Theorem 21. For $r \geqslant 3$, no homogeneous linear equation of at least $r+1$ variables is $r$-graph-regular for $r$-uniform hypergraphs.

Proof. We show the result for $r=3$, and suggest the appropriate modifications for higher $r$.

Assume each $a_{i}$ is nonzero, since discarding trivial variables only makes it easier to be graph-regular.

For any $n$, define an $(n+1)$-coloring $f_{n}^{(3)}$ of $\left(\begin{array}{c}\mathbb{N} \\ r\end{array}\right)$ by

$$
f_{n}^{(3)}(a n+x, b n+y, c n+z)= \begin{cases}\text { blue } & \text { if } x=y=z \\ \min \{x, y, z\} & \text { if one of } x, y, z \text { is smallest } \\ \max \{x, y, z\} & \text { otherwise }\end{cases}
$$

where $x, y, z \in\{0,1, \ldots, n-1\}$. Similar to before, any set of four elements which is monochromatic under this coloring must be blue. 
Now define $g_{n}^{(3)}$ on $\left(\begin{array}{l}\mathbb{N} \\ r\end{array}\right)$ by

$$
g_{n}^{(3)}\left(n^{i} a, n^{j} b, n^{k} c\right)= \begin{cases}f_{n-1}^{(3)}(a, b, c) & \text { if } i=j=k \\ \text { red } & \text { if one of } i, j, k \text { is smallest } \\ \text { green } & \text { otherwise },\end{cases}
$$

where $a, b, c$ are not divisible by $n$. Again, similar to before, any monochromatic clique under this coloring on at least four points must be red or blue. The proofs of Lemmas 8 and 10 now apply essentially unchanged to show that the coefficients of a hypergraphregular equation must sum to zero.

Therefore, we only consider $\sum_{i=1}^{k} a_{i} x_{i}=0$ where $\sum a_{i}=0$.

Define a new coloring, $h_{n}(x, y, z)=g_{n}(y-x, z-x)$, where $x<y<z$, and $g_{n}$ is the graph-coloring used in Section 3.1.

Suppose $x_{1}, \ldots, x_{k}$ are distinct values satisfying $\sum a_{i} x_{i}=0$, with the hyperedges among them monochromatic - either red or blue. Let $x_{j}$ be the smallest of these values. Since $a_{j}=-\sum_{i \neq j} a_{i}$, we see that

$$
\sum_{i \neq j} a_{i}\left(x_{i}-x_{j}\right)=0
$$

By choice of $x_{j}$, we see that $\left\{x_{i}-x_{j}\right\}_{i \neq j}$ is monochromatic under $g_{n}$. As before, a red clique means some $a_{i}$ is 0 . If the clique is blue, then $\sum_{i \neq j} a_{i}=0$, meaning $a_{j}=0$. Since none of the coefficients are 0 , we have reached a contradiction. Thus no homogeneous linear equation in at least 4 variables is hypergraph-regular under colorings of 3 -sets.

For a general $r$-uniform hypergraph with $r>3$, one can easily modify the definition of $g_{n}^{(3)}$ to find a suitable $g_{n}^{(r)}$, which will force coefficients to add to zero. Likewise, one may define a coloring similar to $h_{n}$ which is built upon $g_{n}^{(r-1)}$, which will force one of the coefficients to be zero. These two colorings together will avoid solutions to any equation in at least $r+1$ variables.

Evidently, the ability to color 3-sets (or higher) of integers is too strong to permit monochromatic solutions to homogeneous linear equations. Is there a better definition for an equation to be $r$-graph-regular which allows some equations to meet it, or is this the end of the story?

\section{References}

[1] W. Deuber, D. S. Gunderson, N. Hindman, D. Strauss, Independent finite sums for $K_{m}$-free graphs, J. Combin. Theory Ser. A 78 (1997), no. 2, 171-198.

[2] W. Gasarch, C. Kruskal, A. Parrish, Van der Waerden's theorem and its variants, to appear.

[3] R.L. Graham, B.L. Rothschild, J. Spencer, Ramsey Theory, John Wiley \& Sons Inc., New York, second edition 1990. 
[4] D. S. Gunderson, I. Leader, H. J. Prömel, and V. Rödl, Independent arithmetic progressions in clique-free graphs on the natural numbers, J. Combin. Th. Ser. A 93 (2001), 1-17.

[5] D. S. Gunderson, I. Leader, H. J. Prömel, and V. Rödl, Independent Deuber sets in graphs on the natural numbers, J. Combin. Th. Ser. A 103 (2003), 305-322.

[6] A. Parrish, An additive version of Ramsey's theorem. J. Comb. 2 (2011), no. 4, 593613.

[7] R. Rado, Studien zur Kombinatorik, Math. Zeit. 36 (1933), 242-280.

[8] F. P. Ramsey, On a problem in formal logic, Proc. London Math. Soc. (2), 30 (1930), 264-286. 